Pramana - J. Phys., Vol. 36, No. 6, June 1991, pp. 621-627. (C) Printed in India.

\title{
A comparison of barrier type tunnel junction and point contact tunnel junction formed on the same high $T_{c}$ material
}

\author{
H SRIKANTH and A K RAYCHAUDHURI \\ Department of Physics, Indian Institute of Science, Bangalore 560012, India \\ MS received 5 February 1991; revised 19 March 1991
}

\begin{abstract}
By making a combination of both point contact and barrier type tunnel junctions on a single sample of the high $T_{c}$ superconductor BSCCO (2212) single crystal, we have shown that as the tunneling tip is slowly retracted from the surface a point contact junction gradually evolves from a N-S short to a high resistance tunnel junction. The scaled dynamic conductance $(\mathrm{d} I / \mathrm{d} V$ ) f this point contact tunnel junction becomes almost identical to that of a conventional barrier type tunnel junction and both show a linear $\mathrm{d} I / \mathrm{d} V-V$ curve. The observation implies that at high resistance a point contact junction behaves in the same way as a barrier type tunnel junction. We suggested that the almost linear tunneling conductance obtained in both the cases most likely arises due to an intrinsic characteristic of the surface of the crystal comprising of a mosaic of superconducting regions of the order of a few nanometers. We also conclude that the barrierless $(\mathrm{N}-\mathrm{S})$ point contact obtained by piercing the surface oxide layer of the crystal shows Andreev reflection which we suggest as the origin of the zero bias anomaly often observed in point contact junctions.
\end{abstract}

Keywords. Point contact; tunneling; cryogenic scanning tunneling microscope; Andreev reflection; oxide superconductors.

PACS No. $74 \cdot 50$

\section{Introduction}

Quasiparticle tunneling in point contact and barrier type tunnel junctions has widely been used to study energy gaps in superconductors (Wolf 1985). Particularly with the advent of high $T_{c}$ superconductors, a large number of tunneling studies using both the geometries have been reported (Kirtley 1990; Srinivasan 1990). The native oxide layer on the high $T_{c}$ superconductors has been used in the past to form barrier type tunnel junctions (Cucolo et al 1989). In point contact geometry, the tunnel junctions are generally made by bringing a sharply etched metal tip in close proximity to the sample surface using a cryogenic scanning tunneling microscope (CSTM). Though many studies using both the techniques have been reported, a direct comparison of the data from barrier type tunnel junction and point contact tunnel junction has never been made by doing the experiments on the same material. Very few reports exist on systematic variation of tunneling conductance as a function of junction resistance particularly addressing the issue whether a low resistance point contact curve will gradually evolve at high resistance into a tunneling curve showing the same characteristics as a barrier type tunnel junction (Edgar et al 1987; Qiang Huang et al 1989). The importance of this particular issue is discussed later. In a recent experiment we have addressed this question albeit in an indirect way (Srikanth and Raychaudhuri 1991a). In this communication we report an experimental arrangement by which the 
barrier tunneling (with natural oxide) and the point contact tunneling were done at the same time and on the same crystal. This allowed us to compare these two techniques commonly used for obtaining tunneling data in a direct way.

\section{Experiment}

The experiment was done at $4 \cdot 2 \mathrm{~K}$ on a single crystal $\mathrm{Bi}(2212)\left(T_{c}=-82 \mathrm{~K}\right)$ and $\mathrm{Au}$ was used as the tip material for the point contact. For the barrier type tunnel junction, the natural oxide served as the barrier and $\mathrm{Ag}$ was the counter electrode. In figure 1 we give a schematic of our experiment. The crystal with natural oxide and a layer of $\mathrm{Ag}$ was mounted on a clean $\mathrm{Cu}$ plate which served as the heat sink as well as contact pad to the Ag counter electrode. This forms the barrier type (S-I-N) tunnel junction. The other surface of the crystal was mechanically cleaned and immediately electrodes (conductive Ag pads) attached which served as the two leads of the four probe point contact and tunnel junction. The point contact junction was formed by the Au tip using a CSTM-type arrangement. In this set up, the contact resistance of the point contact junction can be varied in a controlled way and in the same low temperature run simultaneously one can see the conductance $(\mathrm{d} I / \mathrm{d} V-V)$ of the barrier type tunnel junction. The details of the point contact set up and of the electronics are given elsewhere (Srikanth and Raychaudhuri 1991b; Srikanth et al 1991).

In this arrangement (see figure 1) by proper combination of leads we can realize the following situations. With current through $I_{2}$ and $I_{3}$ and the voltage measured across $V_{2}$ and $V_{3}$ we can get the dynamic conductance $(\mathrm{d} I / \mathrm{d} V-V)$ data for the point contact junction. We call this configuration as junction 2-3 for future reference. With $I_{1}$ and $I_{3}$ as the current leads and $V_{1}$ and $V_{3}$ as the potential leads one can measure the dynamic conductance of the barrier type tunnel junction. This is referred to as junction 1-3. When the point contact is pressed hard against the crystal which has a junction resistance much lower than the barrier type tunnel junction, then using $I_{2}$ and $I_{1}$ for current and $V_{2}$ and $V_{1}$ for voltage one can also measure the dynamic conductance of the barrier type tunnel junction. This is called junction 1-2. If junction

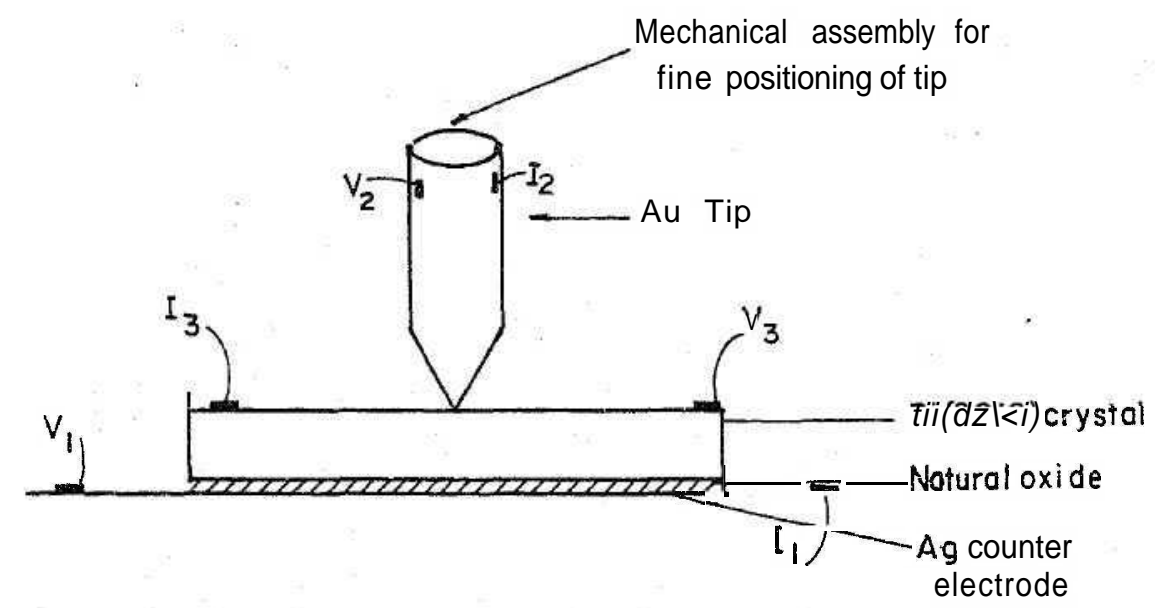

Figure 1. Schematic of the experimental arrangement I's are current leads and $V$ 's are potential leads. Combination 2-3 is used for the point contact junction and combination 1-3 for the natural oxide planar tunnel junction. 
1-3 shows the same characteristics as junction 1-2, then this is an additional evidence that one has achieved a barrierless point contact with the Au tip.

Unless otherwise mentioned, the data were taken by ac modulation technique with modulation amplitude $\simeq 100 \mu \mathrm{V}$. All data were taken at $4 \cdot 2 \mathrm{~K}$ by dipping the sample in liquid helium.

\section{Results and discussion}

In figure 2 we show the dynamic conductance of the point contact (2-3) junction taken at three junction resistances. The zero bias conductances (ZBC) of the junctions shown vary from $55 \mathrm{mS}$ to $39 \mu \mathrm{S}$. The data were normalized at zero bias. One can clearly see that as the tip is moved far away from the material and the junction resistance increases, the dynamic conductance qualitatively changes. At the lowest resistance one sees a very prominent central feature followed by a slowly rising conductance occurring for $\mid h>10-12 \mathrm{mV}$. At higher junction resistance, the central feature is gradually suppressed but distinct gap-like features persist and for junctions with resistance $>1 \mathrm{k} \Omega$ the central feature almost completely vanishes and one observes a linear curve with weak gap-like or phonon-like features occurring at certain characteristicenergies.

The strong features occurring at definite energies in the dynamic conductance curves of the lower junction resistance are special features of $\mathrm{Bi}(2212)$ crystal and are not seen in YBCO (123) single crystals (Srikanth and Raychaudhuri 1991a). Given the limited

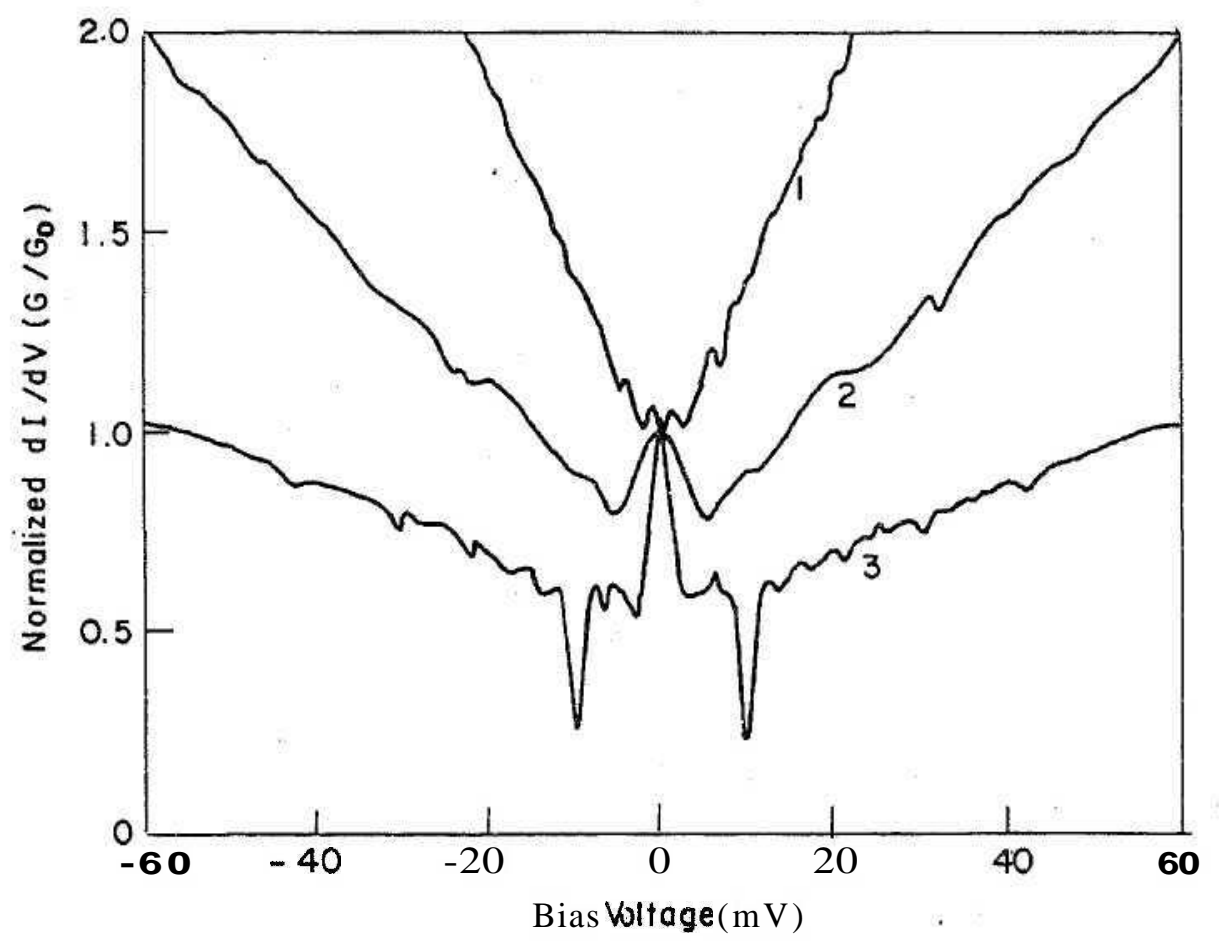

Figure 2. The normalized conductance data $\left(G / G_{0}\right)$ for point contact junctions formed by Au tip on $\operatorname{Bi}(2212)$ single crystal with zero bias conductance $\left(G_{0}\right)$ varying by almost three orders in magnitude. The $G_{0}$ values: $3.9 \times 10^{-5} \mathrm{~S}$ for curve $1,7 \cdot 5 \times 10^{-4} \mathrm{~S}$ for curve 2 and $5 \cdot 5 \times 10^{-2} \mathrm{~S}$ for curve 3 . 
scope of this paper, we do not discuss them here but point out that determination of gap in these materials becomes somewhat ambiguous due to these multiple features.

In figure 3 we show the normalized dynamic conductance of the barrier type tunnel junction (1-3) along with that of the point contact junction (2-3) at high junction resistance $(R>100 \mathrm{k} \Omega)$. Figure 3 very clearly shows that the point contact junction of high resistance and the barrier type tunnel junction show almost identical relative dynamic conductances as a function of bias and both of them have $\mathrm{G} \simeq G_{0}+\alpha \mathrm{K}$ with $\alpha \simeq 2 \times 10^{-7} \mathrm{~S} / \mathrm{mV}$. We think that this similarity of data obtained by two methods is a very important observation and the implications are discussed below. The experiment shows that at high resistance a point contact tunnel junction behaves in the same way as the barrier type tunnel junction (in both cases one sees a linear $\mathrm{d} / / \mathrm{d} V-V$ curve). We would now like to argue that the observed dynamic conductance is an intrinsic characteristic of the system comprising of the superconductor and the oxide layer on top. In the context of point contact junction, Coulomb blockade (single electron tunneling) at the tip-sample junction can give $\mathrm{G} \propto V$ (van Bentum et al 1988; Shivashankar and Raychaudhuri 1990). However such an effect is most likely ruled out in our point contact junction because our junction does not have sufficient high resistance $(R>1 \mathrm{M} \Omega)$. With this arrangement we were not able to get a stable and noiseless point contact junction with resistance above $1 \mathrm{M} \Omega$. Also we think that in the process of touching the sample our tip crashes resulting in an effectively large junction area i.e. a larger junction capacitance. Since the linear $\mathrm{d} I / \mathrm{d} V-V$ curves have been seen in planar barrier type tunnel junctions also it is almost certain that the dominant effect which gives these linear curves has to come either from the surface oxide or from the underlying superconducting material (first few top layers). A comparison of these data also show that our point contact data at high resistance is

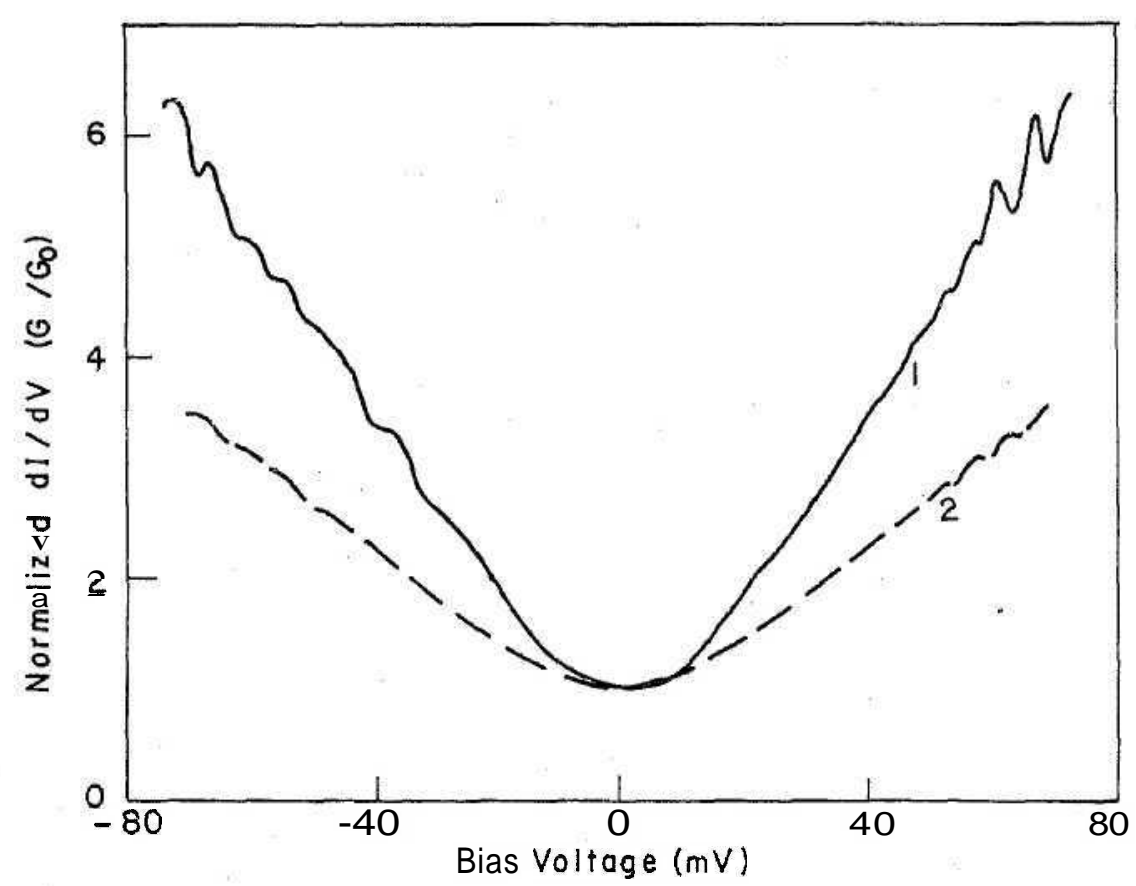

Figure 3. The normalized conductance data $\left(G / G_{0}\right)$ for a high resistance point contact junction (curve $2, G_{0}=0.65 \times 10^{-5} \mathrm{~S}$ ) and a barrier type junction (curve $1, G_{0}=0.24 \times 10^{-5} \mathrm{~S}$ ). Both curves show $\mathrm{G}$ varying linearly with $V$ with very little gap-like or phonon-like features. 
not representative of vacuum tunneling in the proper sense. For junction resistance in the $100 \mathrm{k} \Omega-1 \mathrm{M} \Omega$ range, the tip is most likely within the oxide and the oxide determines the barrier.

In this experiment we made another observation that it is possible to pierce through the surface oxide by the sharp tip. In figure 4 we show the dynamic conductance data for a low resistance point contact junction of $\mathrm{Au}-\mathrm{Bi}(2212)$ along with that of a N-S junction formed by pressing a Pt tip against a $\mathrm{Nb}$ surface. A comparison of the data of $\mathrm{Au}-\mathrm{Bi}(2212)$ and $\mathrm{Pt}-\mathrm{Nb}$ junctions shows that the central feature (i.e. peak in $\mathrm{d} I / \mathrm{d} V-V$ at $\mathrm{F}=0$ ) of the $\mathrm{Au}-\mathrm{Bi}(2212)$ junction of width $\simeq 30 \mathrm{mV}$ arose due to barrierless (or very small barrier) point contact which is formed when the gold tip pierces through the native oxide layer on the $\mathrm{Bi}(2212)$ surface. This is similar to the central peak in $d l / d V$ of the $\mathrm{Pt}-\mathrm{Nb}$ junction of width $\simeq 2-2.5 \mathrm{mV}$. Blonder et al (1982) had studied theoretically barrierless N-S point contact and had shown that $G(0) / G(V>\Delta) \leqslant 2$ for such a junction and this arises due to Andreev reflection. They have also shown that the excess conductance at the zero bias vanishes at $V \geqslant \Delta$. In our case we see the same features in both $\mathrm{Pt}-\mathrm{Nb}$ and $\mathrm{Au}-\mathrm{Bi}(2212)$
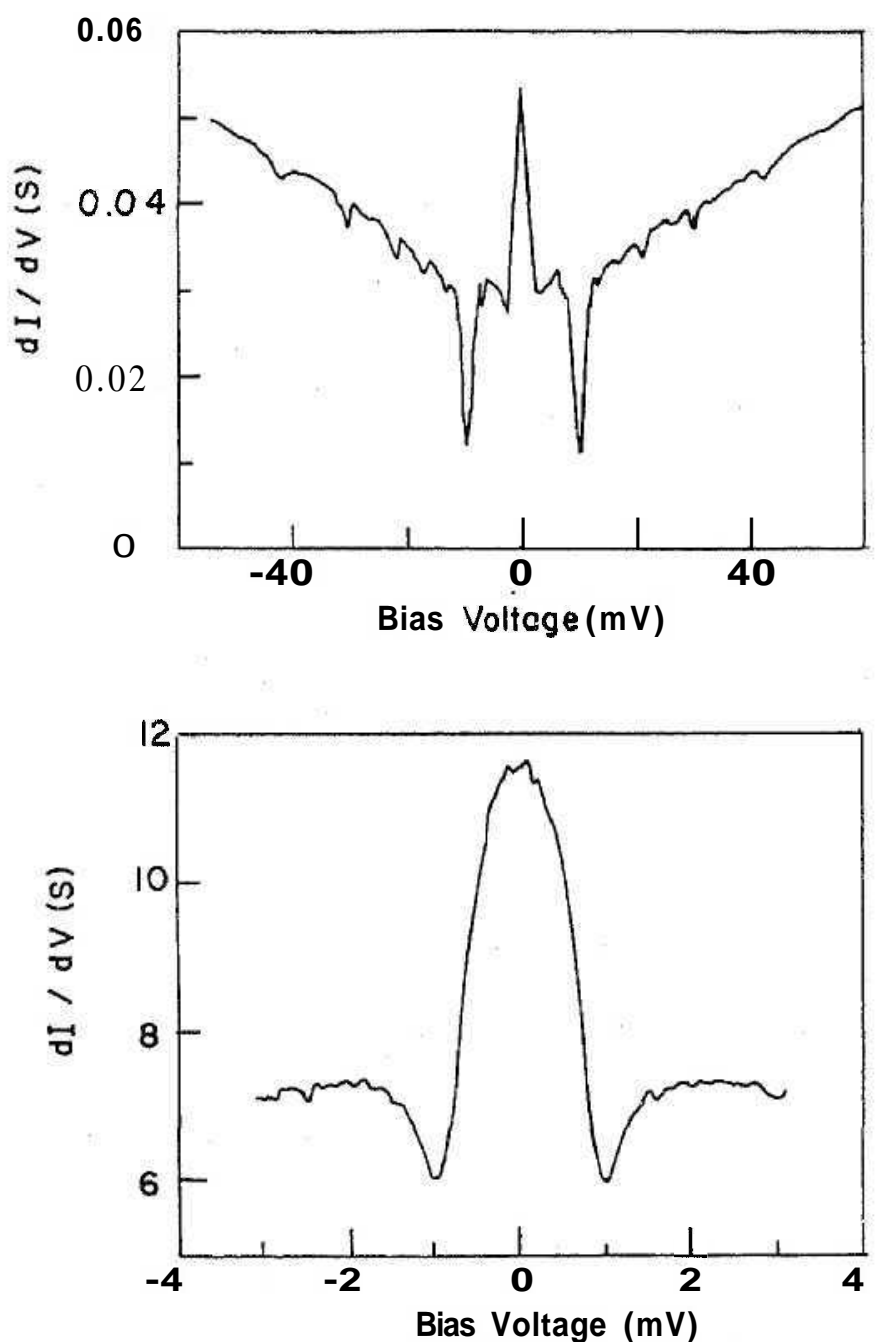

Figure 4. The low resistance, low barrier (or barrierless) N-S type point contacts. Curve A for Au-Bi(2212) and curve B for Pt-Nb junctions. Note the different energy scales of the two junctions. 
junctions. The fact that $\mathrm{G}(0) / \mathrm{G}(V \geqslant \mathrm{~A}) \sim 2$ for both the low resistance junctions implies that the central feature in the dynamic conductance curve arises from Andreev reflection. An alternative can be the tip in intimate contact with the superconductor becoming superconducting by proximity effect and at least a part of the excess conductance at zero bias may be due to Josephson current. Investigation under magnetic field is in progress to settle this issue. There are two differences worth noting between $\mathrm{Pt}-\mathrm{Nb}$ and $\mathrm{Au}-\mathrm{Bi}(2212)$ junctions. Firstly, there is an additional feature occurring within the gap region for the $\mathrm{Au}-\mathrm{Bi}(2212)$ junction which is absent for the $\mathrm{Pt}-\mathrm{Nb}$ junction. Secondly the dynamic conductance is essentially flat for $V>\mathrm{A}$ for the Pt-Nb junction whereas as seen in figure 4 it rises for $\mathrm{Au}-\mathrm{Bi}(2212)$ junction. The explanation for both these is outside the scope of this communication.

At the end we would like to point out a likely scenario for the point contact type junction on high $T_{c}$ oxides and the origin of the linear conductance. The possibility that we can obtain clean $(\mathrm{N}-\mathrm{S})$ point contact on the crystal shows that the surface under the native oxide is superconducting. From the value of the normal state $(V>A)$ junction resistance we estimate using the Sharvin formula (Sharvin 1965) an area of $\simeq 30 \mathrm{~nm}^{2}$. This would imply that at least regions $\leqslant 30 \mathrm{~nm}^{2}$ are homogeneous superconductors. After making a N-S point contact (showing a pronounced central peak) when the tip is gradually retracted one most likely passes through a situation where the junction is a parallel combination of microshort and barrier type junctions. As a result, for junctions with $R \simeq 100 \Omega$ or below one sees the remanence of the central peak as a zero bias anomaly in the conductance curve. In this region the tip receives current from a relatively larger area of the surface. Here we do see gap-like features but a linear conductance background starts developing. Eventually at higher junction resistance the central peak vanishes (implying cessation of N-S microshort behavior) and the point contact junction essentially behaves like a barrier type junction with linear conductance. When the tip is away from the surface it receives current from an extended area. As a likely possibility we suggest that the surface (immediately under the top oxide layer) is inhomogeneous in the scale of a few nanometers. If that is the case, as the tip is moved away it senses current coming from a larger area of the surface. Then the junction will not be like a single S-I-Njunction but more like an "inhomogeneous" or "granular" superconductive tunnel junction and we will get Giaever-Zeller type tunneling leading to a linear conductance curve (Zeller and Giaever 1969). The barrier type tunnel junction spans a much larger area and as a result its conductance will show Giaever-Zeller type behavior. Any attempt to chemically or mechanically remove the native oxide may reconstruct the surface inhomogeneity but it is likely that the inhomogeneity is always there. One observes linear conductance up to $\simeq 100 \mathrm{mV}$ or more. Kirtley et al estimated the size of the spatially homogeneous region to about $10 \mathrm{~nm}$ (Kirtley et al 1987). This is of the same order as 5-6 nm estimated from our N-S point contact data.

Another possibility is that between the top insulating layer and the superconductor there exists a normal conducting metallic layer through which the gap parameter grows from zero to the bulk value in a length scale of a few coherence lengths (Srikanth and Raychaudhuri 1991a). In that case, the tunneling behavior will be due to the normal conducting metallic oxide layer and not the superconducting bulk.

Our experiment cannot, however, distinguish between these two possibilities. Whatever be the case, we feel that the dynamic conductance curves obtained in high resistance junctions do not represent tunneling from or to the bulk superconductor. 


\section{Conclusion}

To conclude, we have shown that by varying the tip-sample separation in a point contact junction it is possible to go continuously from a N-S point contact to a tunneling regime where the linear $\mathrm{d} I / \mathrm{d} V-V$ curve is almost identical to that obtained in a barrier type (natural oxide) planar tunnel junction. Based on our observations, we also suggested a possibility for this linear behavior as arising due to "inhomogeneous" superconductivity of the immediate layer under the natural oxide.

\section{Acknowledgement}

The authors express their thanks to Prof. C N R Rao for giving the Bi(2212) crystal. One of the authors (HS) wishes to thank UGC, Government of India for providing a Research Fellowship.

\section{References}

Blonder G E, Tinkham M and Klapwijk T M 1982 Phys. Rev. B25 4515

Cucolo A M, Valles Jr J M, Dynes R C, Gurvitch M, Phillips J M and Garno J P 1989 Physica C161 351 Edgar A, Adkins C J and Chandler S J 1987 J. Phys. 20 L1009

Kirtley J R 1990 Int. J. Mod. Phys. B4 201

Kirtley J R, Tsuei C C, Park S I, Chi C C, Rozen J and Schafer W M 1987 Phys. Rev. B35 7216

Qiang Huang, Zasadzinski J F, Gray K E, Bukowski E D and Ginsberg D M 1989 Physica C161 141

Sharvin Yu V 1965 Zh. Eksp. Teor. Fiz. 48984 (Sov. Phys. JETP21 655)

Shivashankar G V and Raychaudhuri A K 1990 Pramana - J. Phys. 35 L503

Srikanth H and Raychaudhuri A K 1991a Phys. Rev. B (submitted)

Srikanth H, Rajeswari M and Raychaudhuri A K 199la Pramana-J. Phys. 36207

Srikanth H and Raychaudhuri A K 1991 b Cryogenics (to appear)

Srinivasan R1990 Review article of talk delivered at the discussion meeting on high $T_{\mathrm{f}}$ superconductors, Bangalore August 90

Wolf E L (ed.) 1985 Principles of electron tunneling spectroscopy (Oxford: University Press) van Bentum P J M, van Kempen K, van de Leemput L E C and Teunissan P A A 1988 Phys. Rev. Lett. 60369 Zeller H R and Giaever I 1"969 Phys. Rev. 182789 